\title{
Soft-glass photonic-crystal fibers for frequency shifting and white-light spectral superbroadening of femtosecond Cr:forsterite laser pulses
}

\author{
Andrei B. Fedotov and Dmitry A. Sidorov-Biryukov \\ Department of Physics, International Laser Center, M. V. Lomonosov Moscow State University, Vorob'evy gory, \\ Moscow 119992, Russia \\ Anatoly A. Ivanov and Mikhail V. Alfimov \\ Center of Photochemistry, Russian Academy of Sciences, ul. Novatorov 7a, Moscow 117421, Russia
}

Valentin I. Beloglazov and Nina B. Skibina

Nanostructure Glass Technology Ltd., pr. 50 let Oktyabrya 101, Saratov 410033, Russia

Chi-Kuang Sun

National Taiwan University, Institute of Electro-Optical Engineering, Taipei 10617, Taiwan

Aleksei M. Zheltikov

Department of Physics, International Laser Center, M. V. Lomonosov Moscow State University, Vorob'evy gory, Moscow 119992, Russia

Received August 11, 2005; revised November 21, 2005; accepted December 1, 2005; posted February 3, 2006 (Doc. ID 64024 )

Structural dispersion and nonlinearity management of multicomponent-glass photonic-crystal fibers is shown to allow wavelength-tunable frequency shifting and white-light spectral transformation of femtosecond Cr:forsterite laser pulses. Launching $200 \mathrm{fs}$ pulses of $1.25 \mu \mathrm{m}$ Cr:forsterite laser radiation into such dispersionmanaged soft-glass photonic-crystal fibers in the regime of anomalous dispersion, we demonstrate spectrally tailored supercontinuum generation and frequency upshifting, yielding isolated spectral components with central wavelengths ranging from 400 to $900 \mathrm{~nm}$. (C) 2006 Optical Society of America OCIS codes: $190.4370,190.4360$.

\section{INTRODUCTION}

Photonic-crystal fibers (PCFs) $)^{1,2}$ have proved to be a powerful tool of femtosecond laser technologies, leading to a tremendous enhancement of the capabilities of laser sources of ultrashort pulses and few-cycle field waveforms. Dispersion tunability ${ }^{3}$ and strong field confinement in the fiber core in PCFs ${ }^{4-6}$ are the key factors providing remarkably high efficiencies of nonlinear-optical phenomena, thus allowing the creation of novel fiber-optic broadband light sources ${ }^{7}$ and efficient frequency shifters. ${ }^{8}$ Such PCF components are currently extensively used in many areas of ultrafast science, giving an excess to the carrier-envelope phase, ${ }^{9,10}$ allowing the generation of stabilized frequency combs for optical metrology and femtosecond clockwork, ${ }^{11-13}$ permitting creation of novel optical coherence tomographs ${ }^{14}$ and generators of correlated photons, ${ }^{15}$ simplifying pump-seed synchronization in fewcycle optical parametric chirped-pulse amplifiers, ${ }^{16} \mathrm{im}$ proving the performance of nonlinear spectrographs ${ }^{17}$ and microscopes ${ }^{18}$ based on coherent anti-Stokes Raman scattering, and expanding the applicability range of femtosecond laser sources to photochemistry and photobiology. ${ }^{19}$
Several attractive PCF-based strategies have been demonstrated for highly efficient nonlinear-optical spectral transformation of femtosecond Ti:sapphire laser pulses. These solutions are based on dispersive-wave emission by solitons, ${ }^{20}$ four-wave mixing, ${ }^{21,22}$ modulation instabilities, ${ }^{23}$ stimulated Raman scattering, ${ }^{21}$ and soliton self-frequency shift. ${ }^{24}$ Extension of these strategies of frequency conversion to other input wavelengths and other types of short-pulse sources requires careful PCF dispersion management and sometimes necessitates optimization of the fiber material. Several types of nonsilica PCFs have been used for the spectral transformation of laser radiation in the near-infrared range. An extruded soft-glass SF6 PCF has been used ${ }^{25}$ for ultrabroad supercontinuum generation seeded by $100 \mathrm{fs}$ pulses of $1550 \mathrm{~nm}$ radiation from an optical parametric amplifier. An SF6 PCF was later shown ${ }^{26}$ to allow supercontinuum generation with $200 \mathrm{pJ}$ pulses of $1560 \mathrm{~nm}$ radiation from an allfiber diode-pumped erbium-fiber oscillator-amplifier system used as a pump source. Soliton self-frequency shifts ${ }^{27}$ of mode-locked erbium-fiber laser operating at $1556 \mathrm{~nm}$ have been demonstrated with lead-silicate PCFs. ${ }^{28}$ Phase- 
matched third-harmonic generation by femtosecond $\mathrm{Cr}$ :forsterite laser pulses has been observed in multicomponent-glass PCFs. ${ }^{29}$

We will show in this work that nonsilica PCFs can offer interesting alternatives to silica microstructure fibers for supercontinuum spectral transformation and frequency upshifting of femtosecond pulses within the $1.2-1.3 \mu \mathrm{m}$ range of central wavelengths. This range of wavelengths, covered by femtosecond Cr:forsterite lasers, is of special importance for biomedical imaging. ${ }^{30-32}$ As compared with $800 \mathrm{~nm}$ Ti:sapphire laser radiation, femtosecond pulses of $1.2-1.3 \mu \mathrm{m}$ radiation can provide a significant improvement in penetration depth for rapidly growing nonlinear-optical bioimaging techniques applied to human skin tissues and other biological objects. Already noticeable for the fundamental wavelengths of the Ti:sapphire and Cr:forsterite lasers (800 nm versus $1.25 \mu \mathrm{m})$, this improvement becomes quite dramatic for the second and third harmonics of laser radiation, making a Cr:forsterite laser an advantageous source for bioimaging schemes based on second- and third-harmonic generation, as well as on coherent anti-Stokes Raman scattering. Fiber-optic components for the frequency conversion of $\mathrm{Cr}$ :forsterite laser pulses would thus allow the creation of compact devices for the wavelength-selective imaging of biotissues. We will show in this work that the structural dispersion and nonlinearity management of multicomponent-glass PCFs enables wavelength-tunable frequency shifting and white-light spectral transformation of femtosecond $\mathrm{Cr}$ :forsterite laser pulses. By coupling $200 \mathrm{fs}$ pulses of $1.24 \mu \mathrm{m} \mathrm{Cr}$ :forsterite laser radiation into different types of multicomponent-glass PCFs, where the zero group-velocity dispersion (GVD) wavelength is tuned by scaling the sizes of the fiber structure, we demonstrate spectrally tailored supercontinuum generation and frequency upshifting, yielding isolated spectral components with central wavelengths ranging from 400 to $900 \mathrm{~nm}$.

\section{DISPERSION AND NONLINEARITY OF SOFT-GLASS PHOTONIC-CRYSTAL FIBERS}

Experiments were performed with two types of multicomponent-glass PCFs, fabricated with the use of the standard PCF technology. ${ }^{1,2}$ PCFs of both types were made of multicomponent glass containing approximately $90 \%$ of $\mathrm{S} 93-1$ glass $\left(55 \%\right.$ of $\mathrm{SiO}_{2}, 30 \%$ of $\mathrm{PbO}, 2.0 \%$ of $\mathrm{Al}_{2} \mathrm{O}_{3}, 3.8 \%$ of $\mathrm{Na}_{2} \mathrm{O}$, and $9.2 \%$ of $\mathrm{K}_{2} \mathrm{O}$ ) with additions of S95-2 glass $\left(69.6 \%\right.$ of $\mathrm{SiO}_{2}, 4.0 \%$ of $\mathrm{Al}_{2} \mathrm{O}_{3}, 2.8 \%$ of $\mathrm{B}_{2} \mathrm{O}_{3}$, $6.9 \%$ of $\mathrm{CaO}, 9.0 \%$ of $\mathrm{Na}_{2} \mathrm{O}$, and $7.7 \%$ of $\mathrm{K}_{2} \mathrm{O}$ ). In PCFs of the first type (Fig. 1, top), the solid fiber core is surrounded by a high-air-filling-fraction Kagome-lattice-type cladding. In PCFs of the second type (Fig. 1, bottom), the solid fiber core is surrounded by a single ring of thin-wall capillaries whose outer diameters are equal to the diameter of the PCF core. The outer part of the microstructure cladding consists of 11 rings of capillaries with outer diameters approximately three times larger than the diameter of the PCF core and high air-filling fraction.

Dispersion and nonlinearity of the PCFs of both types were managed by scaling the geometric sizes of the PCF structure. Technologically, this was realized by using the same preform to fabricate PCFs with the same type of the

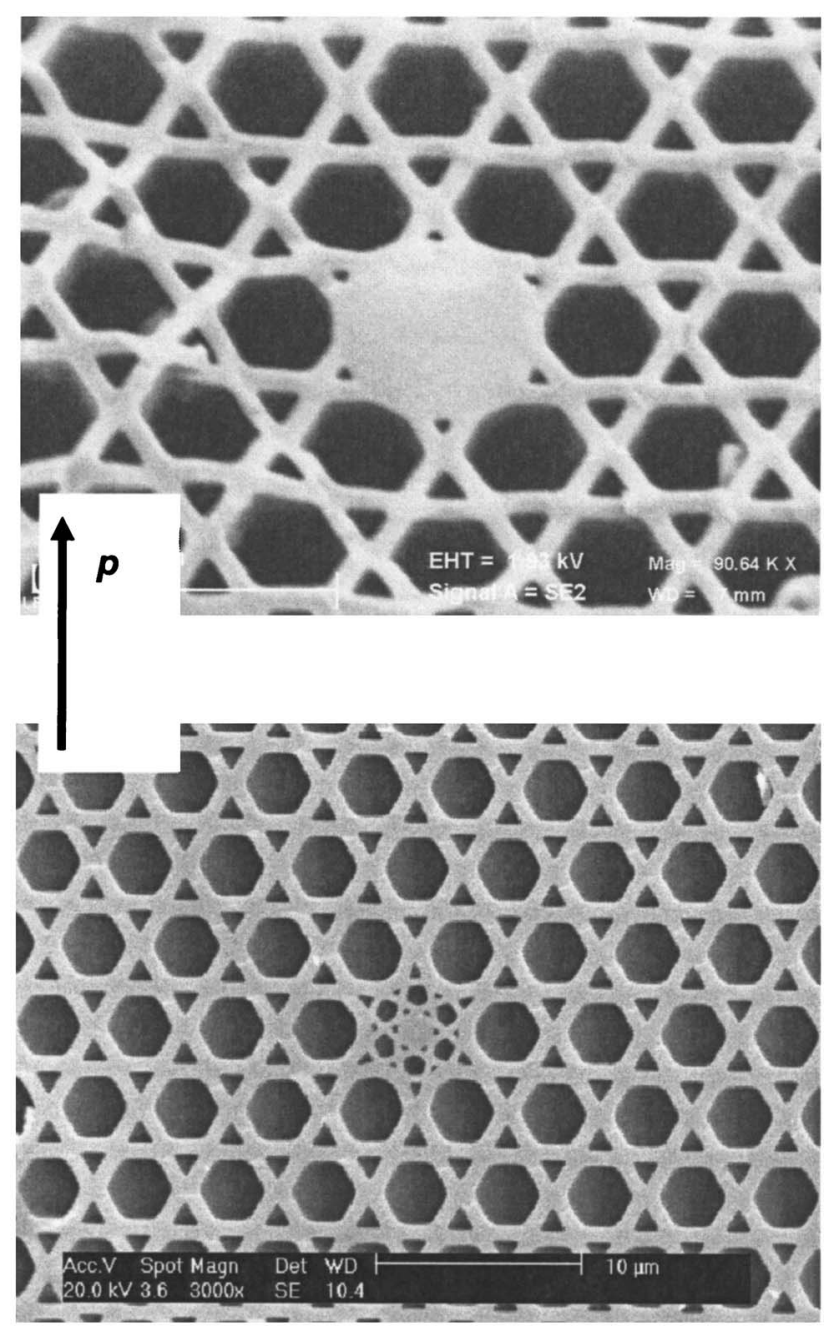

Fig. 1. SEM images of soft-glass photonic-crystal fibers. The $p$ axis shows polarization of the input field.

structure, but with different magnifying factors. This procedure allowed us to scale the sizes of PCF structure without changing its geometry. In Fig. 2, we plot the GVD as a function of the wavelength calculated for the PCF of the first type using the finite-element method, adapted to include the specific fiber structure and material dispersion. As the PCF core diameter is changed from 1.5 to $4 \mu \mathrm{m}$, the zero-GVD wavelength is tuned from 814 to $1175 \mathrm{~nm}$ (curves 1-4 in Fig. 2). For comparison, the material GVD of the multicomponent glass employed to fabricate PCFs is presented by line 5 in Fig. 2. The material part of the GVD passes through zero at $1.66 \mu \mathrm{m}$.

PCFs with smaller cores provide a stronger confinement of guided modes to the fiber core, leading to higher nonlinearity. To quantify this nonlinearity enhancement for the fibers used in this work, we consider the nonlinear coefficient, defined as $\gamma=(2 \pi / \lambda)$ $\times\left[\iint n_{2}(x, y) S_{z}^{2} \mathrm{~d} x \mathrm{~d} y /\left|\iint S_{z} \mathrm{~d} x \mathrm{~d} y\right|^{2}\right]$, where $\lambda$ is the wavelength, $n_{2}(x, y)=n_{2} \xi(x, y), n_{2}$ is the nonlinear refractive index of the fiber material, $\xi(x, y)$ is the two-dimensional spatial profile of the nonlinear refractive index in the PCF cross section, and $S_{z}$ is the $z$ component of the Poynting vector (with the $z$ axis chosen in the direction of waveguiding). In Fig. 2 (top), this nonlinear coefficient is 
plotted as a function of the wavelength $\lambda$ for the PCFs of the first type. More compact modes of PCFs with a smaller core diameter, as can be seen from these plots, are characterized by higher nonlinear coefficients $\gamma$ than modes with larger core diameters. As the radiation wavelength increases, on the other hand, the nonlinearity lowers because of the explicit dependence of $\gamma$ on $\lambda$ and because of the increase in the effective mode area with growing $\lambda$.

To compare dispersion and nonlinear properties of our multicomponent-glass PCFs with the properties of fusedsilica fibers, we also plot in Fig. 2 (bottom, curves 6 and 7) the GVD and the nonlinear coefficient for fibers consisting of a circular fused-silica core and an air cladding with core diameters equal to those of first-type soft-glass PCFs employed in our experiments. With the nonlinear refractive index of our soft-glass PCF, $n_{2} \approx 2.5 \times 10^{-16} \mathrm{~cm}^{2} / \mathrm{W}$, being very close to the $n_{2}$ of fused silica, air-cladded fusedsilica rods provide nonlinear coefficients $\gamma$ close to those of
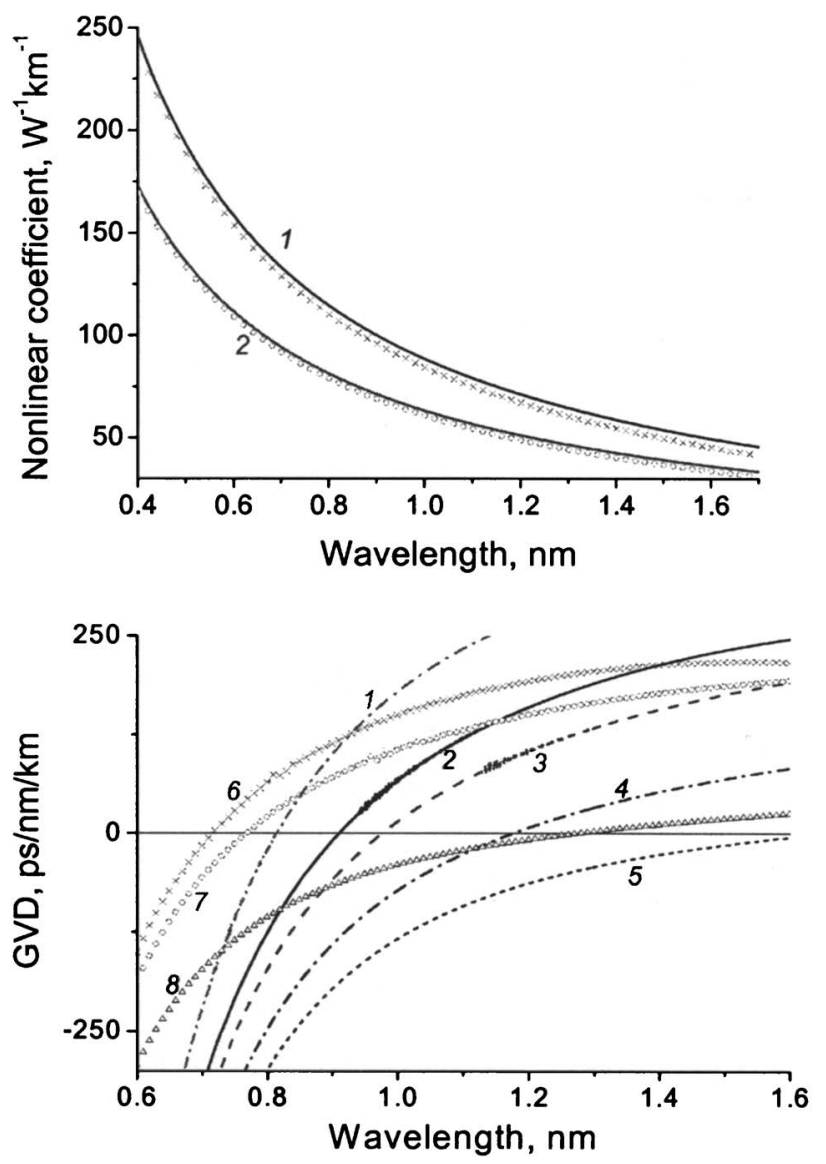

Fig. 2. Bottom, GVD as a function of the wavelength for softglass PCFs with the cross-section structure shown in Fig. 1 (top) and a core diameter of (1) 1.5 , (2) 2, (3) 2.4 , and (4) $4 \mu \mathrm{m}$. Material GVD for PCF glass is shown by curve 5. Also shown is the GVD for a fiber consisting of a circular fused-silica core and an air cladding with a core diameter of (crosses, curve 6) $2 \mu \mathrm{m}$ and (circles, curve 7) $2.4 \mu \mathrm{m}$. Material GVD for silica is shown by curve 8 (triangles) Top, the nonlinear coefficient $\gamma$ as a function of the wavelengths for the same type of PCF with a core diameter of (1) 2 and (2) $2.4 \mu \mathrm{m}$, compared with the nonlinear coefficients for circular fused-silica fibers with an air cladding and a core diameter of (crosses) $2 \mu \mathrm{m}$ and (circles) $2.4 \mu \mathrm{m}$. soft-glass PCFs with equal core diameters (see Fig. 2, top). However, dispersion properties of silica fibers, as can be seen from Fig. 2, drastically differ from those of softglass PCFs. While the GVD of soft-glass PCFs passes through zero at 910 and $980 \mathrm{~nm}$ for core diameters 2 and $2.4 \mu \mathrm{m}$ (curves 2 and 3), respectively, zero GVD for silica rods with the same diameters is achieved at 715 and $767 \mathrm{~nm}$ (curves 6 and 7), respectively. Thus, owing to a different material dispersion (material GVD for silica is shown by curve 8 in Fig. 2, bottom), zero-GVD wavelengths of silica fibers lie much further away from the central wavelength of $\mathrm{Cr}$ :forsterite laser radiation ( $1.25 \mu \mathrm{m}$ in our experiments) than in the case of the softglass PCFs with nearly identical nonlinearities. This comparison suggests that the soft-glass PCFs with the abovespecified parameters are better suited for white-light spectral broadening of Cr:forsterite laser pulses. In particular, dispersion of soft-glass PCFs allows phase matching for Cherenkov-type emission of dispersive waves ${ }^{33,34}$ in the visible by solitons produced by $\mathrm{Cr}$ :forsterite laser pulses. Such phase matching is illustrated by the upper curves in Figs. 3(a) and 3(b), showing the mismatch $\delta$ $=\beta_{s}\left(\lambda_{0}\right)-\beta\left(\lambda_{d}\right)$ of the propagation constant $\beta\left(\lambda_{d}\right)$ of dispersive-wave emission at the wavelength $\lambda_{d}$ in the fundamental mode of the fiber and the propagation constant of the soliton $\beta_{s}\left(\lambda_{0}\right)$ centered at the pump wavelength $\lambda_{0}$ $=1.25 \mu \mathrm{m}$. This resonant emission of dispersive waves, as shown by earlier studies (e.g., Ref. 20) and as confirmed by the results of experiments presented below in this paper, is an important ingredient of supercontinuum generation in PCFs.

Although the nonlinear refractive index $n_{2}$ in our PCFs remained close to that of fused silica, higher $n_{2}$ values can be found in other fiber-technology-compatible materials. Excellent reviews of high- $n_{2}$ glasses and high-Ramangain materials, offering new interesting possibilities in nonlinear fiber optics, have been provided by Friberg and Smith $^{35}$ and Stegeman et al. ${ }^{36}$ As the fiber material is changed, the material dispersion profile is modified as well. In particular, Petropoulos et $a .^{28}$ demonstrated PCFs made of high- $n_{2}$ lead-silicate SF57 glass. The nonlinear refractive index for this glass, $n_{2} \approx 4.1$ $\times 10^{-15} \mathrm{~cm}^{2} / \mathrm{W}$, is more than 1 order of magnitude higher than that for fused silica. The zero-GVD wavelength of bulk SF57 glass is $\sim 1970 \mathrm{~nm}$, i.e., is substantially shifted toward longer wavelength with respect to the zero-GVD wavelength of bulk silica. Zero-GVD wavelengths for PCF modes also tend to be redshifted relative to zero-GVD for identical PCF structures, but made of fused silica. For chalcogenide glasses, the increase in material nonlinearity is even more dramatic, as $n_{2}$ may be as high as 1.1 $\times 10^{-13} \mathrm{~cm}^{2} / \mathrm{W}$ (Ref. 37). In addition to their high nonlinearity and Raman gain, chalcogenide glasses can be transparent for wavelengths up to about $5 \mu \mathrm{m}$, showing promise $^{38}$ for the development of fiber-optic switches and sensors, as well as Raman fiber lasers and amplifiers in the near-, mid-, and long-wave IR spectral regions. Fabrication of chalcogenide PCFs was reported by Monro et al. ${ }^{39}$

The laser system used in our experiments ${ }^{32}$ consisted of a $\mathrm{Cr}^{4+}$ :forsterite master oscillator, a stretcher, an optical isolator, a regenerative amplifier, and a compressor. The 

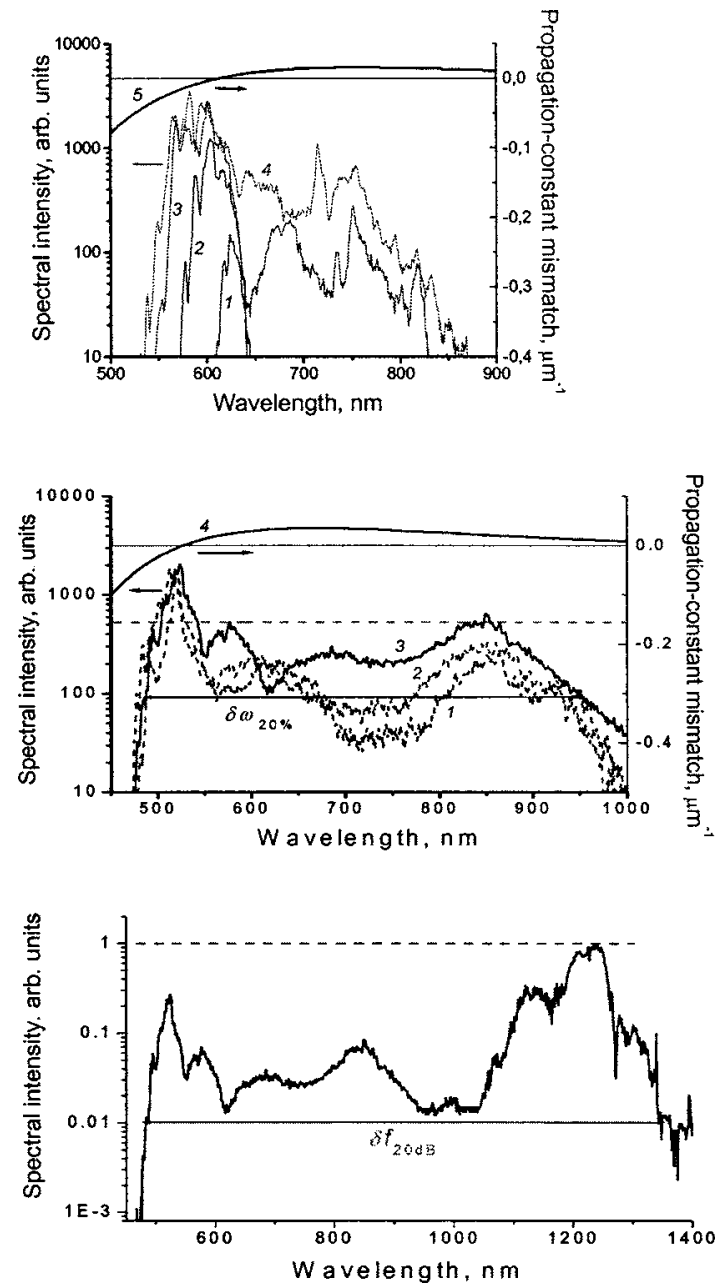

Fig. 3. Output spectra of a soft-glass photonic-crystal fiber with a cross-section structure shown in Fig. 1 (top) and a core diameter of (a) 2.4 and (b) $2 \mu \mathrm{m}$. The input amplified Cr:forsterite laser pulse has a pulse width of $200 \mathrm{fs}$ and an energy of (a) (1) 10, (2) 16, (3) 25, and (4) $40 \mathrm{~nJ}$ and (b) (1) 20, (2) 30, and (3) $40 \mathrm{~nJ}$. The fiber length is $12 \mathrm{~cm}$. Curve 5 in Fig. 3(a) and curve 4 in Fig. 3 (b) show the propagation-constant mismatch $\delta$ between the soliton centered at $1.25 \mu \mathrm{m}$ and dispersive waves guided by the fundamental mode of the PCF as a function of the wavelength of the dispersive wave. The horizontal dashed line in Fig. 3(b) represents the average maximum level formally defined as the halfsum of the amplitudes of the peaks centered at 580 and $860 \mathrm{~nm}$; $\delta \omega_{20 \%}$ is the bandwidth of the output radiation measured at the level of $20 \%$ of the average maximum. (c) The entire radiation spectrum measured at the output of the first-type PCF with a core diameter of $2.0 \mu \mathrm{m}$ and a length of $15 \mathrm{~cm}$. The energy of the input pulse is $32 \mathrm{~nJ} ; \delta \omega_{20} \mathrm{~dB}$ is the width of the total output spectrum measured at the level of $20 \mathrm{~dB}$ of the maximum spectral intensity.

master oscillator, pumped with a fiber ytterbium laser, generated 30-60 fs light pulses of radiation with a wavelength of $1.23-1.25 \mu \mathrm{m}$ at a repetition rate of $120 \mathrm{MHz}$. These pulses were then transmitted through a stretcher and an isolator, to be amplified in a Nd:YLF-laserpumped amplifier and recompressed to the $200 \mathrm{fs}$ pulse duration with the maximum laser pulse energy up to $20 \mu \mathrm{J}$ at $1 \mathrm{kHz}$. The energy of laser pulses used in experiments presented in this paper ranged from 0.5 up to $200 \mathrm{~nJ}$. Because of the slight form anisotropy of the PCF, output radiation spectra were sensitive to the polariza- tion of the input field. Below in this paper, we present experimental results obtained with an input field linearly polarized along the $p$ axis, shown in Fig. 1.

\section{RESULTS AND DISCUSSION}

For both first- and second-type PCFs used in our experiments, the central wavelength of Cr:forsterite laser pulses falls within the range of anomalous dispersion. Such pulses can therefore form solitons in the fiber. Highorder dispersion induces wave-matching resonances between solitons and dispersive waves, ${ }^{33,34}$ giving rise to intense blueshifted emission, observed as prominent features in the PCF output spectra [curves 1-4 in Fig. 3(a) and curves 1-3 in Fig. 3(b)]. The central wavelength of this blueshifted emission correlates well with the wavelength where dispersive waves guided in the fundamental mode of the PCF are phase matched with the soliton produced by Cr:forsterite laser pulses. In particular, our analysis for the first-type PCF with a core diameter of $2.4 \mu \mathrm{m}$ predicts soliton dispersive-wave phase matching around $613 \mathrm{~nm}$ [curve 5 in Fig. 3(a)]. This is exactly where the spectrum of the blueshifted signal is centered for amplified input pulses with energies of 8-12 $\mathrm{nJ}$ transmitted through the first-type PCF with a core diameter of $2.4 \mu \mathrm{m}$ and a length of $12 \mathrm{~cm}$. As the input energy is increased, this component tends to broaden and its highfrequency edge becomes shifted toward shorter wavelengths, owing to the generation of multiple solitons and cross-phase modulation (the influence of cross-phase modulation on supercontinuum generation in PCFs was recently highlighted by Genty et $a l .{ }^{40}$ ), eventually giving rise to a broadband emission at the output of the PCF.

First-type PCFs with a core diameter of $2.0 \mu \mathrm{m}$, as can be seen from Fig. 2 (top), provide a higher nonlinearity than PCFs with a core diameter of $2.4 \mu \mathrm{m}$ do, leading to the generation of broader output spectra for the same input energy and fiber length. Similar to $2.4 \mu \mathrm{m}$ corediameter PCFs, the dominant peak in the output spectra of PCFs with a core diameter of $2.0 \mu \mathrm{m}$ is observed at the wavelength where a dispersive-wave guided mode of the PCF is phase matched with the soliton pump field [as illustrated by curve 4 in Fig. 3(b)]. This resonant dispersive-wave emission gives rise to a peak at 530-540 nm in Fig. 3(b). To quantify spectral broadening, we define the average maximum level of spectral intensity for the output spectra with the largest spectral extension [curve 3 in Fig. 3(b)] as the half-sum of the amplitudes of second most intense peaks in the output spectrum, observed at 580 and $860 \mathrm{~nm}$. This formal definition of the maximum level [shown by the horizontal dashed line in Fig. 3(b)] does not include the most intense, but narrowband dispersive-wave peak at $530 \mathrm{~nm}$. The bandwidth $\delta \omega$ of the output radiation is then measured at the level of $20 \%$ of the average maximum, giving, as shown in Fig. 3, $\delta f_{20 \%}=\delta \omega_{20 \%} / 2 \pi \approx 320 \mathrm{THz}$, with the intensity spectrum stretching from 480 to $970 \mathrm{~nm}$ for an input pulse energy of $40 \mathrm{~nJ}$. The maximum energy of radiation emitted in the $480-970 \mathrm{~nm}$ spectral range under these conditions is 70 pJ. In view of $10 \%$ in-coupling efficiency, this corresponds to a frequency-conversion efficiency of approximately $2 \%$. 
Figure 3(c) displays the entire radiation spectrum measured at the output of the first-type PCF with a core diameter of $2.0 \mu \mathrm{m}$ and a length of $15 \mathrm{~cm}$. Defining the total width $\delta f_{20 \mathrm{~dB}}$ of the output PCF spectrum at the level of $20 \mathrm{~dB}$ below the maximum, we find from Fig. 3(c) that $\delta f_{20 \mathrm{~dB}} \approx 550 \mathrm{THz}$. The output spectrum is dominated by two broad radiation bands, corresponding to the visible emission (from approximately 500 to $930 \mathrm{~nm}$ ) and the spectrally broadened pump (from 1100 to $1320 \mathrm{~nm}$ ). These two bands are separated by a dip in the output spectrum, stretching from approximately 930 to $1100 \mathrm{~nm}$. This dip tends to decrease with the increase in the fiber length, as the spectral widths of both the visible and infrared parts of the output spectrum continue to grow. However, the length of PCF samples in our experiments was limited by fiber losses, estimated as $2-10 \mathrm{~dB} / \mathrm{m}$. With the dominant loss mechanisms in our conditions originating from an incomplete control of glass impurities, an improved fabrication procedure should allow the level of losses for multicomponent-glass PCFs to be reduced to several tens of decibels per kilometer. Although such a level of optical losses will still be substantially higher than the minimum loss levels below decibel per kilometer attainable with fused-silica $\mathrm{PCFs},{ }^{1}$ efficient frequency upconversion provided by multicomponent-glass PCFs presented here, resulting in the generation of intense spectral components in the visible, offers attractive solutions for coherent nonlinear spectroscopy ${ }^{17}$ and microscopy, ${ }^{18}$ as well as for time-resolved spectroscopic measurements. ${ }^{41}$ Because of PCF losses and the low incoupling efficiency (about 10\%), high-input powers were needed in our measurements, requiring the use of a $\mathrm{Cr}$ :forsterite amplifier, for efficient spectral transformations of Cr:forsterite laser pulses. Even with these problems remaining to be resolved, multicomponent-glass PCFs can enhance spectral transformations of Cr:forsterite laser pulses relative to silica PCFs, as the former fibers can simultaneously provide a suitable dispersion regime (Fig. 2) and a small effective mode area, leading to a high nonlinearity for the central wavelength of Cr:forsterite laser radiation (the inset in Fig. 2).

The phase matching between the soliton produced by the input laser pulse and the dispersive wave, which defines the frequency of the dominant peaks in output spectra of PCFs, is controlled by the dispersion of the fiber. The central frequency of the blueshifted signal in the output of PCFs can thus be adjusted by modifying the dispersion profile of the fiber. This option is demonstrated by the results of our experiments performed with secondtype PCFs. The GVD profiles of waveguide modes were modified by scaling the geometric sizes of the fiber without changing the type of the structure shown in Fig. 1 bottom. The core diameter of PCFs fabricated for these experiments was varied from 0.9 to $3.8 \mu \mathrm{m}$. Figure 4 shows the spectra of the blueshifted output of the fiber, adjusted by changing the fiber to vary the offset $\Delta=\lambda_{0}-\lambda_{z}$ between the central wavelength of the input laser field, $\lambda_{0}$, and the zero-GVD wavelength $\lambda_{z}$. With the PCF length remaining unchanged $(10 \mathrm{~cm})$, larger blueshifts are achieved by increasing the offset $\Delta$ (see curves 1-6 in Fig. 4). The power of the input laser field needs to be increased for larger $\Delta$ in these experiments to keep the amplitude of the blue-

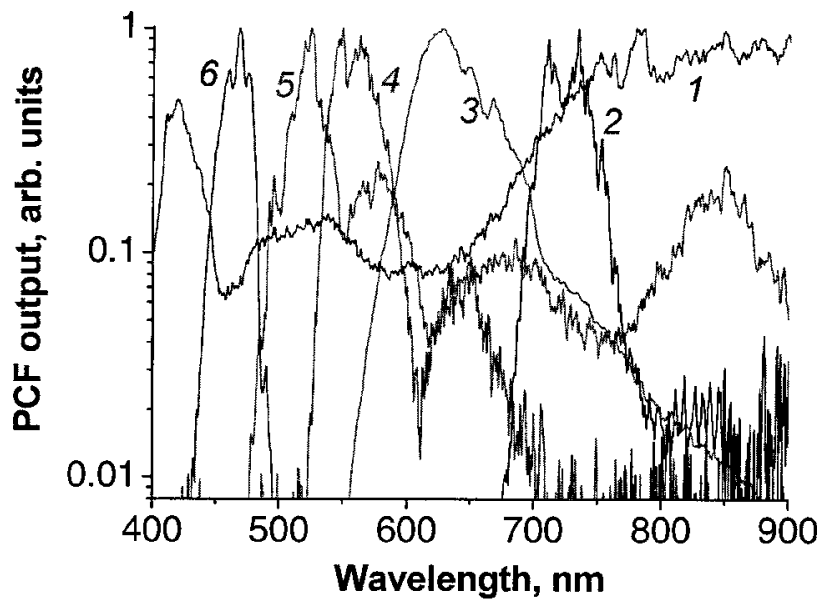

Fig. 4. Intensity spectra of the dispersion-managed blueshifted output of soft-glass PCFs. The wavelength offset $\delta$ is $50 \mathrm{~nm}$ (1), 110 (2), 150 (3), 190 (4), 220 (5), and $300 \mathrm{~nm} \mathrm{(6).}$

shifted signal constant. In these experiments, dispersionmanaged soft-glass PCFs are shown to serve as frequency shifters of femtosecond Cr:forsterite laser pulses, providing an anti-Stokes output adjustable across the range of wavelengths from 400 to $900 \mathrm{~nm}$.

\section{CONCLUSION}

We have shown in this work that the structural dispersion and nonlinearity management of multicomponentglass PCFs allows a wavelength-tunable frequency shifting and white-light spectral transformation of femtosecond Cr:forsterite laser pulses. We experimentally demonstrated frequency shifting and white-light spectral transformation of femtosecond Cr:forsterite laser pulses in nonsilica PCFs. This study allowed us to identify important advantages of multicomponent-glass PCFs over silica microstructure fibers for the spectral transformation of laser pulses in the $1.2-1.3 \mu \mathrm{m}$ spectral range. By coupling $200 \mathrm{fs}$ pulses of $1.24 \mu \mathrm{m} \mathrm{Cr}$ :forsterite laser radiation into different types of multicomponent-glass PCFs, where the zero-GVD wavelength is tuned by scaling the sizes of the fiber structure, we have demonstrated spectrally tailored supercontinuum generation and frequency upshifting, yielding isolated spectral components with central wavelengths ranging from 400 to $900 \mathrm{~nm}$.

\section{ACKNOWLEDGMENTS}

This study was supported in part by the Russian Foundation for Basic Research (projects 06-02-16880 and 05-0290566-NNS), the Russian Federal Research and Technology Program (contract 02.434.11.2010), and INTAS (projects 03-51-5037 and 03-51-5288). The research described in this publication was made possible in part by Award RUP2-2695 from the U.S. Civilian Research and Development Foundation for the Independent States of the Former Soviet Union (CRDF). 


\section{REFERENCES}

1. P. St. J. Russell, "Photonic crystal fibers," Science 299 , 358-362 (2003).

2. J. C. Knight, "Photonic crystal fibers," Nature 424, 847-851 (2003).

3. W. H. Reeves, D. V. Skryabin, F. Biancalana, J. C. Knight, P. St. J. Russell, F. G. Omenetto, A. Efimov, and A. J. Taylor, "Transformation and control of ultra-short pulses in dispersion-engineered photonic crystal fibers," Nature 424, $511-515$ (2003)

4. V. Finazzi, T. M. Monro, and D. J. Richardson, "The role of confinement loss in highly nonlinear silica holey fibers," IEEE Photonics Technol. Lett. 15, 1246-1248 (2003).

5. M. A. Foster, K. D. Moll, and A. L. Gaeta, "Optimal waveguide dimensions for nonlinear interactions," Opt. Express 12, 2880-2887 (2004).

6. A. M. Zheltikov, "The physical limit for the waveguide enhancement of nonlinear-optical processes," Opt. Spectrosc. 95, 410-415 (2003).

7. W. J. Wadsworth, A. Ortigosa-Blanch, J. C. Knight, T. A. Birks, T. P. M. Mann, and P. St. J. Russell, "Supercontinuum generation in photonic crystal fibers and optical fiber tapers: a novel light source," J. Opt. Soc. Am. B 19, 2148-2155 (2002).

8. D. A. Akimov, E. E. Serebryannikov, A. M. Zheltikov, M. Schmitt, R. Maksimenka, W. Kiefer, K. V. Dukel'skii, V. S. Shevandin, and Yu. N. Kondrat'ev, "Efficient anti-Stokes generation through phase-matched four wave mixing in higher-order modes of a microstructure fiber," Opt. Lett. 28, 1948-1950 (2003).

9. D. J. Jones, S. A. Diddams, J. K. Ranka, A. Stentz, R. S. Windeler, J. L. Hall, and S. T. Cundiff, "Carrier-envelope phase control of femtosecond mode-locked lasers and direct optical frequency synthesis," Science 288, 635-639 (2000).

10. A. Baltuska, T. Fuji, and T. Kobayashi, "Self-referencing of the carrier-envelope slip in a 6 -fs visible parametric amplifier," Opt. Lett. 27, 1241-1243 (2002).

11. R. Holzwarth, T. Udem, T. W. Hänsch, J. C. Knight, W. J. Wadsworth, and P. St. J. Russell, "Optical frequency synthesizer for precision spectroscopy," Phys. Rev. Lett. 85, 2264-2267 (2000)

12. S. A. Diddams, D. J. Jones, J. Ye, S. T. Cundiff, J. L. Hall, J. K. Ranka, R. S. Windeler, R. Holzwarth, T. Udem, and T. W. Hänsch, "Direct link between microwave and optical frequencies with a $300 \mathrm{THz}$ femtosecond laser comb," Phys. Rev. Lett. 84, 5102-5105 (2000).

13. D. J. Jones, S. A. Diddams, J. K. Ranka, A. Stentz, R. S. Windeler, J. L. Hall, and S. T. Cundiff, "Carrier-envelope phase control of femtosecond mode-locked lasers and direct optical frequency synthesis," Science 288, 635-639 (2000).

14. I. Hartl, X. D. Li, C. Chudoba, R. K. Rhanta, T. H. Ko, J. G. Fujimoto, J. K. Ranka, and R. S. Windeler, "Ultrahighresolution optical coherence tomography using continuum generation in an air-silica microstructure optical fiber," Opt. Lett. 26, 608-610 (2001).

15. J. G. Rarity, J. Fulconis, J. Duligall, W. J. Wadsworth, and P. St. J. Russell, "Photonic crystal fiber source of correlated photon pairs," Opt. Express 13, 534-544 (2005).

16. C. Y. Teisset, N. Ishii, T. Fuji, T. Metzger, S. Köhler, A. Baltuška, F. Krausz, and A. M. Zheltikov, "All-optical pump-seed synchronization for low-cycle OPCPA," in Conference on Lasers and Electro-Optics, OSA Trends in Optics and Photonics Series (Optical Society of America, 2005).

17. S. O. Konorov, D. A. Akimov, E. E. Serebryannikov, A. A. Ivanov, M. V. Alfimov, and A. M. Zheltikov, "Crosscorrelation FROG CARS with frequency-converting photonic-crystal fibers," Phys. Rev. E 70, 057601 (2004).

18. H. N. Paulsen, K. M. Hilligsøe, J. Thøgersen, S. R. Keiding, and J. J. Larsen, "Coherent anti-Stokes Raman scattering microscopy with a photonic crystal fiber based light source," Opt. Lett. 28, 1123-1125 (2003).

19. S. O. Konorov and A. M. Zheltikov, "Frequency conversion of subnanojoule femtosecond laser pulses in a microstructure fiber for photochromism initiation," Opt. Express 11, 2440-2445 (2003).

20. J. Herrmann, U. Griebner, N. Zhavoronkov, A. Husakou, D. Nickel, J. C. Knight, W. J. Wadsworth, P. St. J. Russell, and G. Korn, "Experimental evidence for supercontinuum generation by fission of higher-order solitons in photonic fibers," Phys. Rev. Lett. 88, 173901 (2002).

21. S. Coen, A. H. L. Chau, R. Leonhardt, J. D. Harvey, J. C. Knight, W. J. Wadsworth, and P. St. J. Russell, "Supercontinuum generation by stimulated Raman scattering and parametric four-wave mixing in photonic crystal fibers," J. Opt. Soc. Am. B 19, 753-764 (2002).

22. J. M. Dudley, L. Provino, N. Grossard, H. Maillotte, R. S. Windeler, B. J. Eggleton, and S. Coen, "Supercontinuum generation in air-silica microstructured fibers with nanosecond and femtosecond pulse pumping," J. Opt. Soc. Am. B 19, 765-771 (2002).

23. J. D. Harvey, R. Leonhardt, S. Coen, G. K. L. Wong, J. C. Knight, W. J. Wadsworth, and P. St. J. Russell, "Scalar modulation instability in the normal dispersion regime by use of a photonic crystal fiber," Opt. Lett. 28, 2225-2227 (2003).

24. X. Liu, C. Xu, W. H. Knox, J. K. Chandalia, B. J. Eggleton, S. G. Kosinski, and R. S. Windeler, "Soliton self-frequency shift in a short tapered air-silica microstructure fiber," Opt. Lett. 26, 358-360 (2001).

25. V. R. K. Kumar, A. K. George, W. H. Reeves, J. C. Knight, P. St. J. Russell, F. G. Omenetto, and A. J. Taylor, "Extruded soft glass photonic crystal fiber for ultrabroad supercontinuum generation," Opt. Express 10, 1520-1525 (2002).

26. H. Hundertmark, D. Kracht, D. Wandt, C. Fallnich, W. R. K. Kumar, A. K. George, J. C. Knight, and P. St. J. Russell, "Supercontinuum generation with 200 pJ laser pulses in an extruded SF6 fiber at $1560 \mathrm{~nm}$," Opt. Express 11, 3196-3201 (2003)

27. G. P. Agrawal, Nonlinear Fiber Optics (Academic, 2001).

28. P. Petropoulos, H. Ebendorff-Heidepriem, V. Finazzi, R. C. Moore, K. Frampton, D. J. Richardson, and T. M. Monro, "Highly nonlinear and anomalously dispersive lead silicate glass holey fibers," Opt. Express 11, 3568-3573 (2003).

29. A. N. Naumov, A. B. Fedotov, A. M. Zheltikov, V. V. Yakovlev, L. A. Mel'nikov, V. I. Beloglazov, N. B. Skibina, and A. V. Shcherbakov, "Enhanced $\chi^{(3)}$ interactions of unamplified femtosecond Cr:forsterite laser pulses in photonic-crystal fibers," J. Opt. Soc. Am. B 19, 2183-2191 (2002).

30. B. Bouma, G. Tearney, I. Bilinsky, B. Golubovic, and J. Fujimoto, "Self-phase-modulated Kerr-lens mode-locked Cr:forsterite laser source for optical coherence tomography," Opt. Lett. 21, 1839-1841 (1996).

31. S. Chu, I. Chen, T. Liu, P. Chen, C. Sun, and B. Lin, "Multimodal nonlinear spectral microscopy based on a femtosecond Cr:forsterite laser," Opt. Lett. 26, 1909-1911 (2001).

32. A. A. Ivanov, M. V. Alfimov, and A. M. Zheltikov, "Femtosecond pulses in nanophotonics," Phys. Uspekhi 47, 687-704 (2004).

33. P. A. Wai, H. H. Chen, and Y. C. Lee, "Radiations by solitons at the zero group-dispersion wavelength of singlemode optical fibers," Phys. Rev. A 41, 426-439 (1990).

34. N. Akhmediev and M. Karlsson, "Cherenkov radiation emitted by solitons in optical fibers," Phys. Rev. A 51, 2602-2607 (1995)

35. S. R. Friberg and P. W. Smith, "Nonlinear optical glasses for ultrafast optical switches," IEEE J. Quantum Electron. 23, 2089-2094 (1987).

36. R. Stegeman, C. Rivero, G. Stegeman, P. Delfyett, Jr., K. Richardson, L. Jankovic, and H. Kim, "Raman gain measurements in bulk glass samples," J. Opt. Soc. Am. B 22, 1861-1867 (2005)

37. R. Slusher, G. Lenz, J. Hodelin, J. Sanghera, L. Shaw, and I. Aggarwal, "Large Raman gain and nonlinear phase shifts in high-purity $\mathrm{As}_{2} \mathrm{Se}_{3}$ chalcogenide fibers," J. Opt. Soc. Am. B 21, 1146-1155 (2004). 
38. P. Thielen, L. Shaw, P. Pureza, V. Nguyen, J. Sanghera, and I. Aggarwal, "Small-core As Se fiber for Raman amplification," Opt. Lett. 28, 1406-1408 (2003).

39. T. M. Monro, Y. D. West, D. W. Hewak, N. G. R. Broderick, and D. J. Richardson, "Chalcogenide holey fibers," Electron. Lett. 36, 1998-2000 (2000).

40. G. Genty, M. Lehtonen, and H. Ludvigsen, "Effect of cross- phase modulation on supercontinuum generated in microstructured fibers with sub-30 fs pulses," Opt. Express 12, 4614-4624 (2004).

41. S. Konorov, A. Ivanov, D. Ivanov, M. Alfimov, and A. Zheltikov, "Ultrafast photonic-crystal fiber light flash for streak-camera fluorescence measurements," Opt. Express 13, 5682-5688 (2005). 